\title{
Impact of the development of an endoscopic eradication program for Barrett's esophagus with high grade dysplasia or early adenocarcinoma on the frequency of surgery*
}

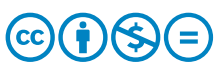

\author{
Authors \\ DeWitt $^{2}$ \\ Institutions \\ 1 Department of Medicine, Indiana University School of \\ Medicine, Indianapolis, IN, USA \\ 2 Division of Gastroenterology, Indiana University School \\ of Medicine, Indianapolis, IN, USA \\ 3 Department of Biostatistics, Indiana University, \\ Indianapolis, IN, USA \\ 4 Department of Surgery, Indiana University School of \\ Medicine, Indianapolis, IN, USA
}

Prianka Chilukuri ${ }^{1,{ }^{* *}}$, Mark A. Gromski ${ }^{2,{ }^{* *}}$, Cynthia S. Johnson ${ }^{3}$, Duy Khanh P. Ceppa ${ }^{4}$, Kenneth A. Kesler ${ }^{4}$, Thomas J. Birdas $^{4}$, Karen M. Rieger ${ }^{4}$, Hala Fatima², William R. Kessler², Douglas K. Rex², Mohammad Al-Haddad², John M.

submitted 8.11.2017

accepted after revision 12.3 .2018

Bibliography

DOI https://doi.org/10.1055/a-0640-3030 |

Endoscopy International Open 2018; 06: E1085-E1092

(c) Georg Thieme Verlag KG Stuttgart · New York

ISSN 2364-3722

Corresponding author

John M. DeWitt, MD, Indiana University School of Medicine, 550 University Blvd, Suite 4100, Indianapolis, IN 46202-

5250, USA

Fax: +1-317-948-8144

jodewitt@iu.edu

\section{ABSTRACT}

Background and aims The impact of the advent of an institutional endoscopic eradication therapy (EET) program on surgical practice for Barrett's esophagus (BE)-associated high grade dysplasia (HGD) or suspected T1a esophageal adenocarcinoma (EAC) is unknown. The aims of this study are to evaluate the different endoscopic modalities used during development of our EET program and factors associated with the use of EET or surgery for these patients after its development.

Methods Patients who underwent primary endoscopic or surgical treatment for BE-HGD or early EAC at our hospital between January 1992 and December 2014 were retrospectively identified. They were categorized by their initial modality of treatment during the first year, and the impact over time for choice of therapy was assessed by multivariable logistic regression.

Results We identified 386 patients and 80 patients who underwent EET and surgery, respectively. EET included single modality therapy in 254 (66\%) patients and multimodal therapy in 132 (34\%) patients. Multivariable logistic regression showed that, for each subsequent study year, EET was more likely to be performed in patients who were older $(P=$ $0.0009)$, with shorter $B E$ lengths $(P<0.0001)$, and with a pretreatment diagnosis of $\operatorname{HGD}(P=0.0054)$ compared to surgical patients. The diagnosis of EAC did not increase the utilization of EET compared to surgery as time progressed $(P=0.8165)$.

Conclusion The introduction of an EET program at our hospital increased the odds of utilizing EET versus surgery over time for initial treatment of patients who were older, had shorter BE lengths or the diagnosis of BE-HGD, but not in patients with EAC.

\footnotetext{
* Abstract and poster presentation: "Primary Endoscopic Therapy vs. Surgical Management of Barrett's Esophagus with High Grade Dysplasia or Early Adenocarcinoma: Experience of a Tertiary Referral Center 1992 2014." DDW 2016, San Diego, CA, USA.

** Co-first authors.
}

\section{Background}

Barrett's esophagus (BE) is the pathologic replacement of esophageal squamous epithelium with goblet cell-lined columnar tissue and intestinal metaplasia [1,2]. A diagnosis of BE is associated with an increased risk for progression to high grade dysplasia (HGD), which is itself associated with a $5-20 \%$ risk of 
esophageal adenocarcinoma (EAC) [3]. EAC is associated with high morbidity and mortality, and its increasing incidence in the United States has prompted targeted screening and surveillance programs for established BE and endoscopic eradication therapies (EET) to treat dysplastic BE and early EAC [3 - 10].

EET has largely replaced surgery as the primary intervention for BE-HGD and early adenocarcinoma (T1a), due to comparable 5-year survival and lower morbidity and adverse events [11-16]. Efficacy and clinical outcomes of individual endoscopic therapies, such as photodynamic therapy (PDT), endoscopic mucosal resection (EMR), radiofrequency ablation (RFA), and cryotherapy are generally similar; however, current therapy with combined EMR and ablation appears to have an increased response to treatment and potentially lower recurrence compared to individual modality treatment alone $[17,18]$. A recent consensus statement noted that EET is an acceptable primary treatment strategy for BE-HGD or early EAC, and that endoscopic intervention is preferred over endoscopic surveillance for such patients [19]. Additionally, EET was incorporated into National Comprehensive Cancer Network (NCCN) guidelines for the primary management of esophageal malignancy in 2012 [20].

Population-based cohort studies suggest that the use of endoscopic therapy to treat BE-HGD and early EAC has increased from 1998 to 2009, with an associated decline in the proportion of surgical cases $[15,21]$. In light of these trends, the impact of such programs on the choice of medical or surgical therapy for individual referral centers remains unknown. With the increasing role of EET in the management of BE-associated dysplasia, we hypothesized that the characteristics of patients treated with esophagectomy at our hospital for these indications would change after the development of an EET program. The primary aim of this study is to evaluate the impact of the development of an EET program on the frequency of primary surgical therapy for treatment-naïve patients with BEHGD and early EAC and to evaluate clinical variables potentially associated with choice of type of therapy in these patients. The secondary aim was to evaluate the evolution of EET modalities used at our center during the study period.

\section{Materials and methods}

\section{Study design and patient selection}

This is a retrospective, single-center study approved by the Institutional Review Board at Indiana University Health Hospital in Indianapolis, Indiana. Utilizing two departmental databases (one surgical database with esophagectomies and one endoscopic database of EET for BE-associated HGD or early EAC), we identified consecutive treatment-naïve patients 18 years or older who underwent either primary endoscopic therapy or surgery for BE-HGD or early EAC (T1a) at our medical center between January 1992 and December 2014. The pretreatment diagnosis of HGD or early EAC was based on the results of initial or reinterpreted endoscopic biopsy specimens, cross-sectional imaging, EUS (if available) and physician assessment. Patients with suspected T1b or higher tumor $(\mathrm{T})$ stage cancers or those previously treated with endoscopic ablation or resection, che- motherapy, radiation, or previous esophageal surgery before initial endoscopic or surgical procedures at our hospital were excluded. The following information was extracted from the medical record: age, gender, race, highest degree of pathologic dysplasia before EET or surgery, pathology of resected specimens post-EET or surgery, preprocedural endoscopic ultrasound (EUS), primary therapeutic modality (EET in the first 12 months of therapy or surgery) performed, and components of the Charlson Comorbidity Index (CCI). Primary EET was further subcategorized as PDT, EMR, RFA, cryotherapy alone, or combination endoscopic therapy based on treatments given during the initial 12 months of EET.

\section{Development of Barrett's EET program}

At our institution before 1998, esophagectomy was the primary modality offered for patients requiring definitive therapy for dysplastic BE or early EAC. Beginning in 1998, we commenced offering PDT for treatment of HGD and, later, for early cancer. This was followed in 2001 by the introduction of cap-assisted and, later, band-ligation assisted EMR. Additional therapies including RFA and cryotherapy, alone or in combination with EMR, were introduced after commercial release (both RFA and cryotherapy introduced in 2007 at our institution). During the study period, all endoscopic therapy was performed by one of six endoscopists specializing in the endoscopic treatment of Barrett's esophagus, and esophagectomies were performed by one of five thoracic surgeons. Decisions on the management approach for each patient were at the discretion of the treating and/or consulting physician. A multidisciplinary conference or protocol-based management at our hospital was not used for these patients, yet referrals were made between gastroenterology and surgery as deemed appropriate by the treating physi$\operatorname{cian}(\mathrm{s})$.

Pathologic interpretation of all biopsies showing HGD from outside institutions was confirmed by review from an expert pathologist at our medical center before initiation of EET. Biopsies of patients diagnosed with cancer were not routinely reconfirmed at our institution. All patients with cancer underwent cross-sectional imaging to confirm absence of metastases; EUS was performed selectively in these patients at the discretion of the treating physician.

\section{Data analysis}

Descriptive statistics consisted of means \pm standard deviations or medians with interquartile ranges for continuous variables, and simple proportions for dichotomous variables. Comparisons between treatment groups (EET vs. surgery) without adjustment for other factors (single-variable analyses) were performed using independent two-sample $t$ tests for continuous variables (age, length of Barrett's esophagus, and Charlson Comorbidity Index) and chi-squared tests for categorical variables (sex, race, pre-therapy EUS, and initial diagnosis). To explore factors associated with selection of initial treatment (BE-HGD vs. EAC), multivariable logistic regression analyses were performed. Year of initial treatment and initial pathologic diagnosis were included in the model. In order to determine whether the initial pathologic diagnosis affected the choice of treat- 
- Table 1 Baseline characteristics of Endoscopic Eradication Therapy (EET) and Surgery groups for the treatment of BE-HGD or early EAC from 1998 to 2014 .

\begin{tabular}{|c|c|c|c|}
\hline & EET $(n=386)$ & Surgery $(n=67)$ & $P$ value \\
\hline Male, n (\%) & $322(83.4)$ & $55(82.1)$ & 0.7880 \\
\hline Caucasian, n (\%) & $379(99.0)$ & $67(100.0)$ & 0.4008 \\
\hline Age, mean $\pm S D$, years & $67.2 \pm 10.8$ & $61.5 \pm 10.1$ & $<0.0001$ \\
\hline Pretreatment EUS, n (\%) & $220(57.0)$ & $59(88.1)$ & $<0.0001$ \\
\hline Charlson Comorbidity Index (CCI) with age, mean \pm SD & $3.9 \pm 2.3$ & $3.6 \pm 2.0$ & 0.33 \\
\hline Pretreatment diagnosis of adenocarcinoma, $\mathrm{n}(\%)$ & $102(26.4)$ & $43(64.2)$ & $<0.0001$ \\
\hline Length of $\mathrm{BE}$, mean $\pm \mathrm{SD}, \mathrm{cm}^{1}$ & $4.2 \pm 3.6$ & $6.8 \pm 3.4$ & $<0.0001$ \\
\hline Post-therapy adenocarcinoma diagnosis², n (\%) & $99(30.1)$ & $43(68.3)$ & $<0.0001$ \\
\hline Agreement between pre- and post-therapy diagnosis, $n(\%)^{3}$ & $176(53.5)$ & $47(74.6)$ & $<0.0001$ \\
\hline \multicolumn{4}{|c|}{$\begin{array}{l}\text { BE-HGD, Barrett's esophagus with high grade dysplasia; EAC, esophageal adenocarcinoma; EUS, endoscopic ultrasound. } \\
1 \text { Data not available for all patients. } \\
2 \text { Post-therapy adenocarcinoma is a pathologic specimen of adenocarcinoma from surgical resection specimen or endoscopic resection specimen/biopsies post-EET } \\
\text { therapy. } \\
{ }^{3} \text { Comparison between the highest grade of pathology from pre-therapy specimens and post EET biopsies/specimens or surgical resection specimens in the surgical } \\
\text { group. }\end{array}$} \\
\hline
\end{tabular}

ment differently in earlier than in later years, the interaction between the treatment year and the initial diagnosis was also included in the model. Additionally, all significant baseline variables with $P<0.25$ in the single-variable analyses were included as covariates. Backwards selection was used to determine the final model. Since the association between the length of Barrett's esophagus with treatment in the single-variable analyses was statistically significant but this variable was not reported in 144 patients, two multivariable logistic regression models were fit: one excluding and one including length of Barrett's esophagus. Since the results of both models were similar, only the results of the full model including length of Barrett's esophagus are presented. All $P$ values $<0.05$ were considered statistically significant and $95 \%$ confidence limits were reported. Statistical analyses were performed using SAS 9.4 (Cary, North Carolina, United States).

\section{Results}

At our institution between January 1992 and December 1997, 13 patients underwent primary surgery for BE-HGD or early EAC. Subsequently, between January 1998 and December 2014, our hospital treated 453 consecutive treatment-naïve patients with surgical ( $n=67)$ or endoscopic therapy $(n=386)$ for BE-HGD or T1a EAC ( $\vee$ Table 1 ). The EET group was older (67.2 vs. 61.5 years, $P<0.0001)$, had fewer diagnoses of esophageal adenocarcinoma before therapy initiation (26.4 vs. $64.2 \%, P<$ $0.0001)$, and completed fewer pretreatment EUS exams ( 57.0 vs. $88.1 \%, P<0.0001)$. Among the 309 (68.2\%) patients with available data, mean BE length was shorter in the EET group compared to the surgery group ( 4.2 vs. $6.8 \mathrm{~cm}, P<0.0001)$. Mean CCls were similar between the EET and surgery groups (3.9 vs. 3.6, $P=0.33$ ). Clinical characteristics of surgery patients from 1992 to 1997 (pre-EET introduction) were similar to sur-
- Table 2 Primary endoscopic $(n=386)$ or surgical $(n=80)$ therapy used during the first 12 months of treatment for 466 patients with Barrett's esophagus high grade dysplasia (BE-HGD) or early esophageal adenocarcinoma (EAC) from 1992 to 2014.

\begin{tabular}{|c|c|c|c|}
\hline \multicolumn{2}{|c|}{$\begin{array}{l}\text { Primary endoscopic therapy } \\
\text { - initial } 12 \text { months }(n=386)\end{array}$} & \multicolumn{2}{|c|}{$\begin{array}{l}\text { Primary surgical therapy } \\
(\mathrm{n}=\mathbf{8 0})\end{array}$} \\
\hline \multicolumn{4}{|c|}{ Single modality therapy, n (\%) } \\
\hline - PDT alone & $45(11.7 \%)$ & $\begin{array}{l}\text { Ivor Lewis } \\
\text { (transthoracic) }\end{array}$ & $36(45.0 \%)$ \\
\hline - EMR alone & $196(50.8 \%)$ & Transhiatal & $20(25.0 \%)$ \\
\hline - RFA alone & $13(3.4 \%)$ & Three incision & $23(28.8 \%)$ \\
\hline $\begin{array}{l}\text { - Cryotherapy } \\
\text { alone }\end{array}$ & $0(0)$ & Unknown & $1(1.3 \%)$ \\
\hline \multicolumn{4}{|c|}{ Combination endoscopic therapy, n (\%) } \\
\hline - $E M R+R F A$ & $83(21.5 \%)$ & & \\
\hline - $E M R+P D T$ & $29(7.3 \%)$ & & \\
\hline - Other ${ }^{1}$ & $20(5.2 \%)$ & & \\
\hline \multicolumn{4}{|c|}{$\begin{array}{l}\text { PDT, photodynamic therapy; EMR, endoscopic mucosal resection; RFA, } \\
\text { radiofrequency ablation. } \\
\text { ' Other = Combined modalities include: EMR + RFA + Cryotherapy, EMR + PDT } \\
\text { + RFA or EMR + Cryotherapy. }\end{array}$} \\
\hline
\end{tabular}

gery patients treated between January 1998 and December 2014 (all $P>0.19$; data not shown).

- Table 2 demonstrates the endoscopic and surgical treatments employed within the study period. Of the 80 surgeries, Ivor-Lewis esophagectomy was performed in $45 \% \quad(n=36)$, while transhiatal and three incision approaches were employed in $25 \%(n=20)$ and $28.8 \%(n=23)$, respectively. The operation performed in one patient (1.2\%) was unknown. EET (index 
- Table 3 Frequency of primary endoscopic $(n=386)$ or surgical $(n=80)$ therapy used by year for 466 consecutive patients from 1992 to 2014 with BE-HGD or T1a EAC.

\begin{tabular}{|c|c|c|c|c|c|c|c|c|c|c|c|}
\hline \multirow[t]{3}{*}{ Year } & \multicolumn{5}{|c|}{ T1a } & \multicolumn{5}{|c|}{ HGD } & \multirow{3}{*}{$\begin{array}{l}\text { All patients } \\
\text { Total } \\
\mathbf{n}\end{array}$} \\
\hline & \multicolumn{2}{|c|}{ Surgery } & \multicolumn{2}{|c|}{ EET } & \multirow{2}{*}{$\begin{array}{l}\text { Total } \\
\mathbf{n}\end{array}$} & \multicolumn{2}{|c|}{ Surgery } & \multicolumn{2}{|c|}{ EET } & \multirow{2}{*}{$\begin{array}{l}\text { Total } \\
\text { n }\end{array}$} & \\
\hline & n & $\%$ & n & $\%$ & & $\mathbf{n}$ & $\%$ & $\mathbf{n}$ & $\%$ & & \\
\hline 1992 & 1 & 100.0 & 0 & 0.0 & 1 & 0 & - & 0 & - & 0 & 1 \\
\hline 1993 & 0 & - & 0 & - & 0 & 1 & 100.0 & 0 & 0.0 & 1 & 1 \\
\hline 1994 & 0 & - & 0 & - & 0 & 0 & - & 0 & - & 0 & 0 \\
\hline 1995 & 2 & 100.0 & 0 & 0.0 & 2 & 1 & 100.0 & 0 & 0.0 & 1 & 3 \\
\hline 1996 & 2 & 100.0 & 0 & 0.0 & 2 & 2 & 100.0 & 0 & 0.0 & 2 & 4 \\
\hline 1997 & 2 & 100.0 & 0 & 0.0 & 2 & 2 & 100.0 & 0 & 0.0 & 2 & 4 \\
\hline 1998 & 0 & 0.0 & 3 & 100.0 & 3 & 2 & 22.2 & 7 & 77.8 & 9 & 12 \\
\hline 1999 & 1 & 25.0 & 3 & 75.0 & 4 & 2 & 28.6 & 5 & 71.4 & 7 & 11 \\
\hline 2000 & 2 & 40.0 & 3 & 60.0 & 5 & 3 & 15.8 & 16 & 84.2 & 19 & 24 \\
\hline 2001 & 0 & 0.0 & 5 & 100.0 & 5 & 1 & 20.0 & 4 & 80.0 & 5 & 10 \\
\hline 2002 & 0 & 0.0 & 5 & 100.0 & 5 & 2 & 25.0 & 6 & 75.0 & 8 & 13 \\
\hline 2003 & 3 & 60.0 & 2 & 40.0 & 5 & 1 & 11.1 & 8 & 88.9 & 9 & 14 \\
\hline 2004 & 5 & 55.6 & 4 & 44.4 & 9 & 3 & 20.0 & 12 & 80.0 & 15 & 24 \\
\hline 2005 & 3 & 16.7 & 15 & 83.3 & 18 & 1 & 6.3 & 15 & 93.7 & 16 & 34 \\
\hline 2006 & 0 & 0.0 & 8 & 100.0 & 8 & 2 & 6.1 & 31 & 93.9 & 33 & 41 \\
\hline 2007 & 1 & 5.9 & 16 & 94.1 & 17 & 0 & 0.0 & 29 & 100.0 & 29 & 46 \\
\hline 2008 & 3 & 25.0 & 9 & 75.0 & 12 & 2 & 9.5 & 19 & 90.5 & 21 & 33 \\
\hline 2009 & 3 & 37.5 & 5 & 62.5 & 8 & 1 & 5.9 & 16 & 94.1 & 17 & 25 \\
\hline 2010 & 8 & 66.7 & 4 & 33.3 & 12 & 0 & 0.0 & 25 & 100.0 & 25 & 37 \\
\hline 2011 & 5 & 55.6 & 4 & 44.4 & 9 & 3 & 9.4 & 29 & 90.6 & 32 & 41 \\
\hline 2012 & 4 & 30.8 & 9 & 69.2 & 13 & 1 & 3.6 & 27 & 96.4 & 28 & 41 \\
\hline 2013 & 3 & 37.5 & 5 & 62.5 & 8 & 0 & 0.0 & 12 & 100.0 & 12 & 20 \\
\hline 2014 & 2 & 50.0 & 2 & 50.0 & 4 & 0 & 0.0 & 18 & 100.0 & 18 & 22 \\
\hline
\end{tabular}

treatment and all endoscopic therapies in the first 12 months) included 254 (66\%) single modality therapies. Single modality treatments included PDT in $11.7 \%$, EMR in $50.8 \%$, and RFA in $3.4 \%$. Combination EET was employed in 132 (34\%) patients.

- Table 3 presents the type of therapy performed (surgery or EET), on an annualized basis by pretreatment pathology (EAC or HGD). As the table illustrates, the highest numbers of patients treated were in 2007 when 46 patients were treated and in 2006, 2011, and 2012 when 41 patients were treated. The table also illustrates a relatively stable proportion of patients with $\mathrm{T} 1 \mathrm{a} \mathrm{EAC}$ undergoing surgery during the last 10 years of the study (2005-2014, with $29.4 \%$ of patients with T1a EAC undergoing surgery during that time). During the same period of time, however, the number of patients undergoing surgery for BE-HGD significantly decreased. > Fig.1 graphically illus- trates the distribution of patients undergoing either surgery or EET on an annualized basis. > Fig. 2 demonstrates the annual percentage contribution of single or combination modality endoscopic treatments to the total EET utilization. Overall, patients underwent a median of 2 (IQR $1-3)$ EET procedures in the initial 12 months of endoscopic therapy. PDT was the first EET modality adopted and was incorporated in all EET cases in 1998 and 1999 but was not used after 2007. EMR was later introduced with significant utilization starting in 2001, and increasing in use either as a sole modality or combination modality thereafter. RFA was first introduced in 2007, predominantly as a combination therapy. Since 2007, the use of EMR and RFA for combination therapy has risen, and in the latter few years of the study, was used in over half of the EET cases. 


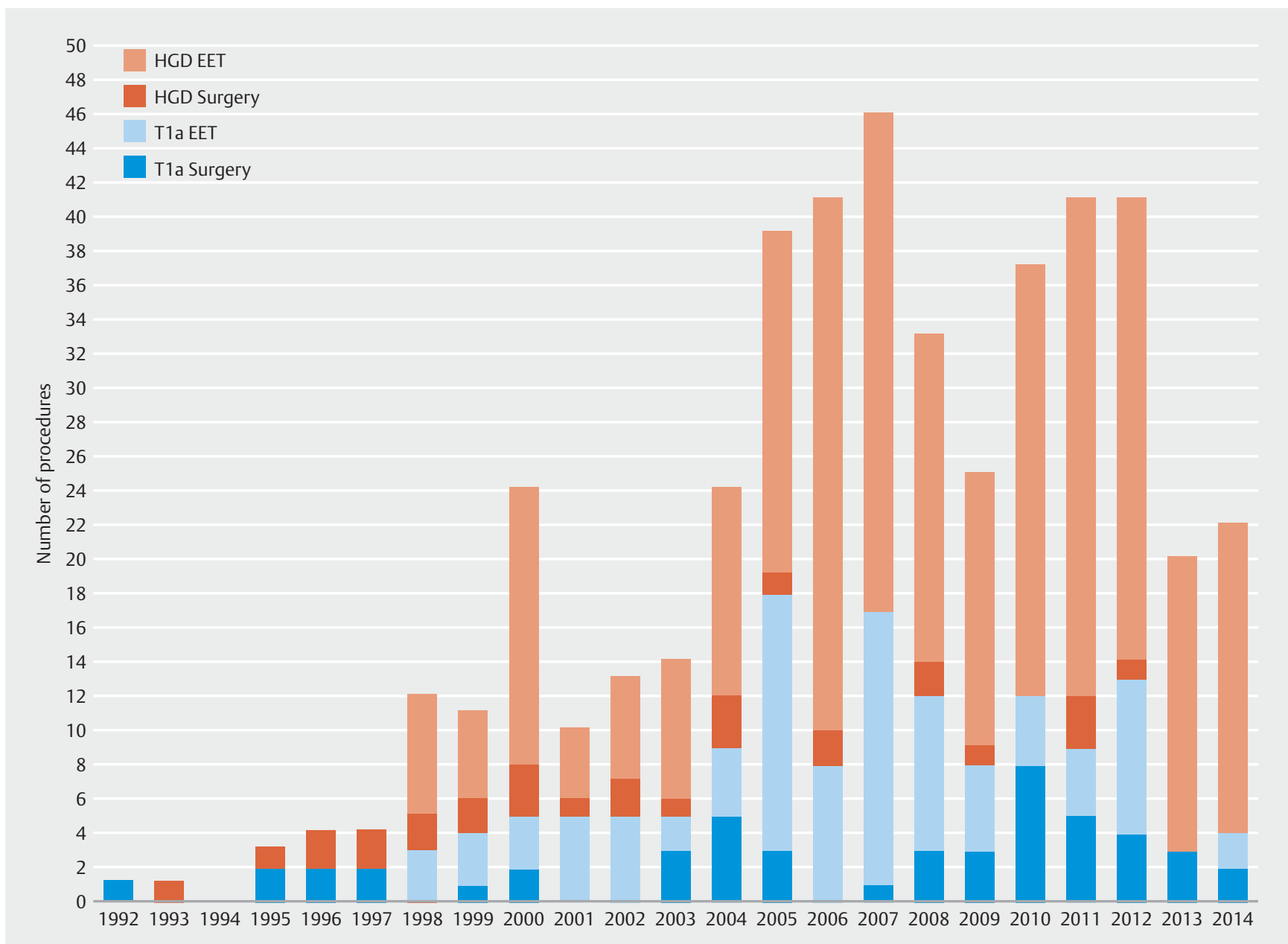

- Fig. 1 Primary endoscopic $(n=386)$ or surgical $(n=80)$ therapy used during the first 12 months of treatment for 466 patients with Barrett's Esophagus with High Grade Dysplasia (BE-HGD) or early esophageal adenocarcinoma (EAC) from 1992 to 2014, displayed on an annualized basis according to therapy performed and underlying pathology.

Single-variable analyses of patients undergoing EET $(n=386)$ or surgery ( $n=67$ ) from 1998 to 2014 showed only age, use of EUS, and length of Barrett's esophagus were significant $(P<$ $0.25)$. Therefore, the multivariable model included these three variables as well as the initial diagnosis (BE-HGD vs. EAC), year of the initial therapeutic procedure, and the interaction term between year of the procedure and initial diagnosis. The multivariable model indicated that older patients were more likely to undergo EET $(P=0.0009)$. The odds of EET compared to surgery increased by $1.22, \mathrm{Cl}(1.09-1.37)$ for every 3-year increase in age; $1.39, \mathrm{Cl}(1.15-1.69)$ for every 5 -year increase in age; and $1.94, \mathrm{Cl}(1.31-2.86)$ for every 10 -year increase in age. Shorter length of BE was significantly associated with EET $(P<0.0001)$. For every $1 \mathrm{~cm}$ decrease in length of $\mathrm{BE}$, the odds of undergoing EET compared to surgery increased by $1.21, \mathrm{Cl}(1.10-1.33)$. The year the exam was performed affected the selected procedure differently by initial diagnosis $(P=0.0182)$. The diagnosis of EAC did not increase the utility of EET compared to surgery as time progressed ( $P=0.8165)$; however, patients initially diagnosed with BE-HGD had higher odds of receiving EET compared to surgery for each subsequent year $(P=0.0054)$. The odds of undergoing EET increased by 1.32 (Cl 1.09-1.62) for each subsequent year ( $\vee$ Table 4$)$.

\section{Discussion}

EET has been incorporated into the National Comprehensive Cancer Network (NCCN) guidelines as a primary modality for the management of early (Tis, T1a) esophageal malignancy at low risk for lymph node metastases [19]. However, about 25 years ago, endoscopic treatment was not generally considered to be a first line treatment for these patients. As with any new modality, incorporation of novel technology and treatment options into consensus guidelines requires demonstration of equivalent or superior but safe outcomes to currently available techniques. A meta-analysis of seven retrospective cohort studies published between 2003 and 2011 showed that, compared to esophagectomy, EET for Barrett's esophagus with HGD or T1a EAC is associated with fewer major adverse events and similar rates of neoplastic remission and 1-year, 3-year, and 5-year survival $[11-13,16,22-25]$. This efficacy is reflected in the 2012 American Society for Gastrointestinal Endoscopy (ASGE) 


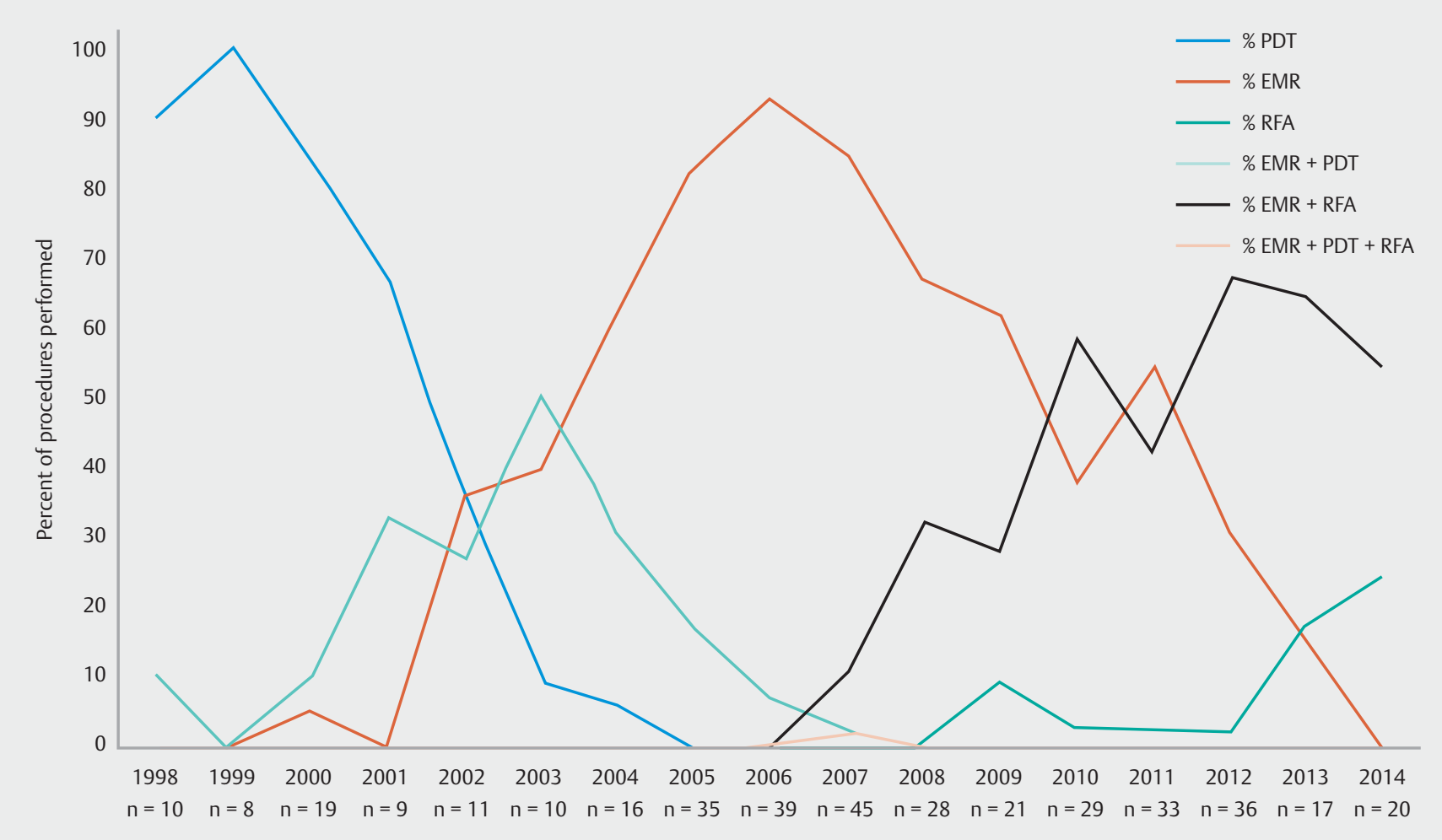

Fig. 2 Annual percentage contribution of single or combination modality endoscopic treatments to the total EET utilization from 1998 to 2014.

- Table 4 Results of Multivariable Logistic Regression analyses comparing factors associated with receiving Endoscopic Eradication Therapy (EET) vs. Surgery from 1998 to 2014.

\begin{tabular}{|c|c|c|c|}
\hline & \multicolumn{3}{|l|}{ EET vs. Surgery } \\
\hline & Odds Ratio (95\% Cl) & Relative Risk (95\% Cl) & $P$ value \\
\hline Age & & & 0.0009 \\
\hline - 3-year increase & $1.22(1.09,1.37)$ & $1.18(1.07,1.29)$ & \\
\hline - 5-year increase & $1.39(1.15,1.69)$ & $1.30(1.12,1.51)$ & \\
\hline - 10-year increase & $1.94(1.31,2.86)$ & $1.67(1.24,2.17)$ & \\
\hline EUS not performed & $4.78(1.24,18.41)$ & $2.90(1.19,4.62)$ & 0.0230 \\
\hline Length of $B E$ ( $1 \mathrm{~cm}$ decrease) & $1.21(1.10,1.33)$ & $1.17(1.08,1.26)$ & $<0.0001$ \\
\hline Initial diagnosis by year & & & 0.0182 \\
\hline - Year for BE-HGD & $1.32(1.09,1.62)$ & $1.25(1.07,1.46)$ & 0.0054 \\
\hline - Year for EAC & $0.98(0.85,1.14)$ & $0.98(0.87,1.11)$ & 0.8165 \\
\hline
\end{tabular}

guidelines for EET of Barrett's associated dysplasia which considered PDT, RFA, cryotherapy, EMR, and endoscopic submucosal dissection (ESD) as viable treatment options in these patients, and EMR or ESD for nodular dysplasia [26]. These options in patients with early cancer were different from the 2003 guidelines in the management of esophageal cancer, which recommended surgical resection for all patients with early esoph- ageal adenocarcinoma who were surgical candidates [27]. In an updated 2013 ASGE publication on the same topic, multiple endoscopic treatment modalities including EMR, ESD, PDT, and RFA were included for management options of early esophageal adenocarcinoma [28].

Similar to the evidence-based evolution of guidelines, our group's therapeutic offerings for these patients have changed. 
Our study highlights the evolution and use of different endoscopic modalities for EET at our hospital following the institution of an EET program in 1998. Before 1998, esophagectomy was the only modality offered at our hospital for management of dysplastic BE or early EAC. Our EET program started in 1998 with PDT monotherapy for BE-HGD and eventually progressed to offer EMR and multimodal therapy for these patients. While the use of PDT waned, EMR was gradually adopted as the principal single modality therapy and was eventually used in combination with RFA for the majority of patients.

In the current study, EET patients were older, had fewer preprocedure diagnoses of esophageal adenocarcinoma, completed fewer pretreatment EUS exams, and had a shorter BE length compared to the surgery group. The more frequent use of EUS in the surgery group is likely explained by the more frequent diagnosis of cancer in this population and the decreasing use over time of EUS in patients diagnosed with HGD alone. Multivariable regression analysis found that patients who were older and had BE-HGD were more likely to undergo EET compared to surgery as study time progressed. Patients with shorter BE length were also more likely to undergo EET; however, the diagnosis of cancer did not affect the choice of treatment received. These findings demonstrate increased use of EET over time at our hospital in patients with BE-HGD but not with suspected T1a EAC.

Although other smaller studies have described institutional approaches to BE-HGD and early EAC over time [14,29], this is the first study to systematically review surgical and endoscopic management at a single institution over two decades. The current study highlights the development of our EET program and investigates several factors which may have impacted the use of EET or surgery for these patients over a 22-year period. However, our study does have some limitations. First, although patients evaluated were chosen from two prospective databases, it is possible that some receiving surgery or endoscopy during the study period were not enrolled. Second, our study did not use a multidisciplinary clinic or protocol to triage these patients to surgical or endoscopic therapy. Factors such as patient preferences, referral patterns, and referring provider recommendations which may have influenced management decisions were not evaluated in the current study.

In conclusion, this single-center retrospective study of the treatment-naïve patient with BE-HGD or early EAC from 1992 to 2014 found that patients who were older, had a pretreatment diagnosis of HGD, and shorter BE length were more likely to undergo EET compared to surgery as study time progressed. Pretreatment diagnosis of T1a esophageal cancer did not affect the choice of treatment received.

\section{Acknowledgements}

This project was IRB approved, protocol number \#1501401098. We would like to acknowledge Jonathan Pike for his contributions in compiling data for the esophagectomy database.
Competing interests

None

References

[1] Spechler SJ. Clinical practice. Barrett's esophagus. NEJM 2002; 346: 836-842 [doi:10.1056/NEJMcp012118]

[2] Spechler SJ, Souza RF. Barrett's esophagus. NEJM 2014; 371: 836 845 [doi:10.1056/NEJMra1314704]

[3] Rastogi A, Puli S, El-Serag HB et al. Incidence of esophageal adenocarcinoma in patients with Barrett's esophagus and high-grade dysplasia: a meta-analysis. Gastrointest Endosc 2008; 67: 394-398 [doi:10.1016/j.gie.2007.07.019]

[4] Begg CB, Cramer LD, Hoskins W] et al. Impact of hospital volume on operative mortality for major cancer surgery. JAMA 1998; 280: $1747-1751$

[5] Swisher SG, Deford L, Merriman KW et al. Effect of operative volume on morbidity, mortality, and hospital use after esophagectomy for cancer. J Thorac Cardiovasc Surg 2000; 119: 1126 - 1132 [doi:10.1067/mtc.2000.105644]

[6] Pohl H, Welch HG. The role of overdiagnosis and reclassification in the marked increase of esophageal adenocarcinoma incidence. J Natl Cancer Inst 2005; 97: 142 - 146 [doi:10.1093/jnci/dji024]

[7] Thrift AP, Whiteman DC. The incidence of esophageal adenocarcinoma continues to rise: analysis of period and birth cohort effects on recent trends. Ann Oncol 2012; 23: 3155 - 3162 [doi:10.1093/annonc/mds181]

[8] Brown LM, Devesa SS, Chow WH. Incidence of adenocarcinoma of the esophagus among white Americans by sex, stage, and age. J Natl Cancer Inst 2008; 100: 1184 - 1187 [doi:10.1093/jnci/djn211]

[9] Eloubeidi MA, Mason AC, Desmond RA et al. Temporal trends (19731997 ) in survival of patients with esophageal adenocarcinoma in the United States: a glimmer of hope? Am J Gastroenterol 2003; 98: 1627 - 1633 [doi:10.1111/j.1572-0241.2003.07454.x]

[10] Conio M, Cameron AJ, Romero Y et al. Secular trends in the epidemiology and outcome of Barrett's oesophagus in Olmsted County, Minnesota. Gut 2001; 48: 304-309

[11] Pech O, Bollschweiler E, Manner $\mathrm{H}$ et al. Comparison between endoscopic and surgical resection of mucosal esophageal adenocarcinoma in Barrett's esophagus at two high-volume centers. Ann Surg 2011; 254: 67 - 72 [doi:10.1097/SLA.0b013e31821d4bf6]

[12] Prasad GA, Wang KK, Buttar NS et al. Long-term survival following endoscopic and surgical treatment of high-grade dysplasia in Barrett's esophagus. Gastroenterology 2007; 132: 1226-1233 [doi:10.1053/j.gastro.2007.02.017]

[13] Prasad GA, Wu TT, Wigle DA et al. Endoscopic and surgical treatment of mucosal (T1a) esophageal adenocarcinoma in Barrett's esophagus. Gastroenterology 2009; 137: 815-823 [doi:10.1053/j.gastro.2009.05.059]

[14] Schmidt HM, Mohiuddin K, Bodnar AM et al. Multidisciplinary treatment of T1a adenocarcinoma in Barrett's esophagus: contemporary comparison of endoscopic and surgical treatment in physiologically fit patients. Surg Endosc 2016; 30: 3391 - 3401 [doi:10.1007/ s00464-015-4621-z]

[15] Wani S, Drahos ], Cook MB et al. Comparison of endoscopic therapies and surgical resection in patients with early esophageal cancer: a population-based study. Gastrointest Endosc 2014; 79: 224-232 e221 [doi:10.1016/j.gie.2013.08.002] 
[16] Wu J, Pan YM, Wang TT et al. Endotherapy versus surgery for early neoplasia in Barrett's esophagus: a meta-analysis. Gastrointest Endosc 2014; 79: 233 - 241 e232 [doi:10.1016/j.gie.2013.08.005]

[17] Barnes JA, Willingham FF. Endoscopic management of early esophageal cancer. J Clin Gastroenterol 2015; 49: 638 - 646 [doi:10.1097| MCG.0000000000000353]

[18] Pouw RE, Wirths K, Eisendrath P et al. Efficacy of radiofrequency ablation combined with endoscopic resection for Barrett's esophagus with early neoplasia. Clin Gastroenterol Hepatol 2010; 8: 23-29 [doi:10.1016/j.cgh.2009.07.003]

[19] Bennett C, Vakil N, Bergman J et al. Consensus statements for management of Barrett's dysplasia and early-stage esophageal adenocarcinoma, based on a Delphi process. Gastroenterology 2012; 143: 336 - 346 [doi:10.1053/j.gastro.2012.04.032]

[20] NCCN. NCCN Clinical Practice Guidelines in Oncology (NCCN Guidelines): Esophageal and Esophagogastric Junction Cancers. Version 2.2012. www.NCCN.org

[21] Ngamruengphong S, Wolfsen HC, Wallace MB. Survival of patients with superficial esophageal adenocarcinoma after endoscopic treatment vs surgery. Clin Gastroenterol Hepatol 2013; 11: 1424-1429 e1422, quiz e1481 [doi:10.1016/j.cgh.2013.05.025]

[22] Zehetner J, DeMeester SR, Hagen JA et al. Endoscopic resection and ablation versus esophagectomy for high-grade dysplasia and intramucosal adenocarcinoma. J Thorac Cardiovasc Surg 2011; 141: $39-$ 47 [doi:10.1016/j.jtcvs.2010.08.058]
[23] Schembre DB, Huang JL, Lin OS et al. Treatment of Barrett's esophagus with early neoplasia: a comparison of endoscopic therapy and esophagectomy. Gastrointest Endosc 2008; 67: 595-601 [doi:10.1016/j.gie.2007.08.042]

[24] Reed MF, Tolis G Jr., Edil BH et al. Surgical treatment of esophageal high-grade dysplasia. Ann Thorac Surg 2005; 79: 1110 - 1115 [doi:10.1016/j.athoracsur.2004.09.006]

[25] Pacifico RJ, Wang KK, Wongkeesong LM et al. Combined endoscopic mucosal resection and photodynamic therapy versus esophagectomy for management of early adenocarcinoma in Barrett's esophagus. Clin Gastroenterol Hepatol 2003; 1: $252-257$

[26] Evans JA, Early DS, Fukami N et al. The role of endoscopy in Barrett's esophagus and other premalignant conditions of the esophagus. Gastrointest Endosc 2012; 76: 1087 - 1094 [doi:10.1016/j. gie.2012.08.004]

[27] Jacobson BC, Hirota W, Baron TH et al. The role of endoscopy in the assessment and treatment of esophageal cancer. Gastrointest Endosc 2003; 57: $817-822$

[28] Evans JA, Early DS, Chandraskhara V et al. The role of endoscopy in the assessment and treatment of esophageal cancer. Gastrointest Endosc 2013; 77: 328 - 334 [doi:10.1016/j.gie.2012.10.001]

[29] O'Farrell N], Reynolds JV, Ravi N et al. Evolving changes in the management of early oesophageal adenocarcinoma in a tertiary centre. Ir ] Med Sci 2013; 182: 363 - 369 [doi:10.1007/s11845-012-0890-x] 